G513(P) DEVELOPING A FRAMEWORK OF STAFF SUPPORT ACROSS A PAEDIATRIC HOSPITAL: THE SUPPORT (SUPPORTING PAEDIATRIC STAFF, PATIENTS AND FAMILIES TO BE ONE RESILIENT TEAM) PROGRAMME

CP Macaulay, H Conniff. General Paediatrics, Evelina London Children's Hospital, London, UK

10.1136/archdischild-2020-rcpch.437

Aims Paediatrics is one of the most diverse and rewarding fields of medicine but also brings with it unique medical and ethical challenges. We also care for increasingly complex medical patients and are faced with ethical dilemmas that can lead to conflict with families and a challenging work environment: it can be emotionally exhausting. At our tertiary children's hospital we became aware that this was having a negative impact on our staff's wellbeing and ability to care for our patients. In particular we recognised that staff were being exposed to distressing events and then expected to continue with clinical work with no formal system to debrief or acknowledge the impact of these events. We aimed to do something to address this.

Methods We established a working group of senior nurses, executive members, Paediatricians and Psychologists to identify issues and implement change through our SuPPORT programme. Within this, we created a staff support psychologist (PSS) role (0.3 WTE) to establish a psychological debrief pathway, coordinate and run debriefs and establish/scope other supports e.g. reflective practice.

Results The group has created a staff support 'pyramid', developing new resources and bringing together activities which were already in place. This includes a robust debrief pathway following serious incidents, and Paediatric Schwartz rounds. The PSS role has been central in this process. Since the role began we have run regular reflective practice sessions in several areas, and held 71 psychology mediated debriefs. Feedback from staff has been hugely positive.

Conclusion By establishing this programme we have acknowledged that staff may feel overwhelmed by the complex medical and ethical situations in paediatric care. We are still in the early stages, but staff report feeling a different 'culture' of support at work. Crucial to this has been the commitment by our senior leadership team. We recognise that these problems are not unique to our hospital and have had interest from other hospitals in spreading this model.

\section{G514(P) EVALUATION OF BURNOUT IN PAEDIATRIC STAFF: A PREVALENCE STUDY}

F Astill, S Harris, H Lewis. Paediatrics, Royal Gwent Hospital, Newport, UK

\subsection{6/archdischild-2020-rcpch.438}

Introduction Burnout in healthcare staff has been highlighted as a concern within the NHS, as it can compromise patient safety, lead to patient and staff dissatisfaction and decreased staff retention.

Method Paediatric staff, including clinical and administrative staff, across Wales completed an anonymous questionnaire to assess their risk of burnout using a validated tool the 'Oldenburg Burnout Inventory'. Free text comments were used to investigate individuals' perceptions of risks and protective factors. Participants were asked to assess their own happiness at work using a Likert scale. This was disseminated by lead consultants and ward managers within the local hospitals.

Results 234 paediatric staff members completed the questionnaire, of which 81 were doctors. Paediatric nursing staff compromised 92 participants.

$68.8 \%$ of paediatric staff scored at high risk of developing burnout. Health care assistants had the greatest proportion at high risk with $94.7 \%$ of staff surveyed.

Doctors in training, including foundation doctors were the least happy occupational group with $6.1 / 10$ with consultants scoring highest at $6.8 / 10$. 1

It was interesting to note that a high self-reported happiness score did not necessarily equate to a low risk of burnout.

$60.4 \%$ said 'no' or 'only a little' when asked if they looked forward to coming to work.

Protective factors were having supportive managers and colleagues with emphasis on meeting regularly. Barriers were environmental factors, workforce shortages and lack of team cohesion.

Conclusion Paediatric staff in Wales are at high risk of developing burnout. Previous research has shown that a systematic approach encompassing individual and organisational interventions can reduce this risk. A pilot wellbeing scheme is being trialled a local hospital, with a focus on team building, wellbeing resources and management engagement for organisational change.

\section{G515(P) 'IN SPITE OF THE GAPS' - A MULTIFACETED APPROACH TO STAFFING A CHILDREN'S HOSPITAL}

${ }^{1} \mathrm{E}$ Evans, ${ }^{1} \mathrm{E}$ Day, ${ }^{2} \mathrm{~A}$ Newnham, ${ }^{1} \mathrm{~V}$ Acharya, ${ }^{1} \mathrm{~S}$ Abrahams, ${ }^{1} \mathrm{~J} H$ Haigh, ${ }^{1} \mathrm{H}$ Duncan, ${ }^{3} \mathrm{~F}$ Campbell. 'Department of Paediatric Medicine, Leeds Children's Hospital, Leeds, UK; ${ }^{2}$ Department of Paediatric Nephrology, Leeds Children's Hospital, Leeds, UK; ${ }^{3}$ Department of Paediatric Diabetes, Leeds Children's Hospital, Leeds, UK

\subsection{6/archdischild-2020-rcpch.439}

The RCPCH UK workforce census made recommendations to ensure sufficient paediatric staffing in the future. Our Children's Hospital has embraced this model and taken a new approach to staffing that enables the provision of high quality, safe paediatric care, with a focus on paediatric training.

We have experienced significant gaps in the trainee medical workforce in recent years. Gaps are spread equitably across the region, however a $20 \%$ gap is usual. Trainees and consultants alike have felt the impact, resulting in concerns about morale, retention and burnout. The effect of receiving yet another email asking for locum cover, or informing you of out of hour's gaps, should not be underestimated.

We adopted a new approach to forecasting and managing the rota gaps. This involves the management team, rota coordinators, college tutors, trainees and medical deployment. A monthly strategic workforce meeting is held, alongside a biweekly meeting to manage day-to-day issues and on-going recruitment.

Recruitment looks 6-12 months ahead - essential when recruiting overseas doctors. We have recruited to 1-2 year posts through NHS jobs, MTI schemes and a trust-wide international recruitment programme. There is bespoke induction and a supernumerary period before starting in ST1-3 rotas. Trainees then move into ST4-8 rotas after 6 months. We support those applying for paediatric training, with anticipated longer-term gains for the regional workforce. 